Article

\title{
Resonant Effects in a Photoproduction of Ultrarelativistic Electron-Positron Pairs on a Nucleus in the Field of the X-ray Pulsar
}

\author{
Nikita R. Larin *(D), Sergei P. Roshchupkin $(\mathbb{D}$ and Victor V. Dubov \\ Department of Theoretical Physics, Peter the Great St. Petersburg Polytechnic University, Polytechnicheskaya 29, \\ 195251 Saint-Petersburg, Russia; serg9rsp@gmail.com (S.P.R.); dubov@spbstu.ru (V.V.D.) \\ * Correspondence: nikita.larin.spb@gmail.com
}

Received: 15 July 2020; Accepted: 29 August 2020; Published: 2 September 2020

check for updates

\begin{abstract}
The resonant photoproduction of the electron-positron pairs on a nucleus near a surface of the X-ray pulsar was studied theoretically. The main feature of the processes, which are responsible for the formation of the electron-positron fluxes, is a capability to occur in a resonant way in the electromagnetic field of the X-ray pulsar. One of the properties of the resonant case is that the initial process of second order in the fine structure constant in an external field effectively reduces into two successive processes of the first order due to the fact that in the resonant conditions intermediate virtual electron (positron) becomes a real particle. It is shown that the resonances are possible only when the initial gamma quantum energy is more than the threshold energy, which significantly depends on the number of absorbed photons of an external electromagnetic field. Additionally, in the resonant conditions, the energies of the particles depend on the outgoing angle of a positron (channel A) or an electron (channel B). It is shown that the resonant differential cross section has an extremely large magnitude in units $\alpha Z^{2} r_{e}^{2}$. A mechanism to explain the presence of anomalous fluxes of ultrarelativistic positrons near the surface of an X-ray pulsar was proposed.
\end{abstract}

Keywords: external X-ray field; Oleinik's resonances; X-ray pulsar; gamma quantum; nucleus

\section{Introduction}

Astroparticle physics has proclaimed itself in previous decades as a dynamically developing and relevant field of investigation due to the fact that it represents the compound of particle physics, astrophysics, and cosmology [1]. Consequently, quantum processes make an essential impact on the evolution of astrophysical objects, for example, such as pulsars and magnetars. The presence of the strong electromagnetic field influences on the run of quantum processes, which are responsible for the composition of the winds from the pulsar surface [2]. Additionally, it is a well-known phenomenon that the cosmic rays contain an anomalous abundance of high-energy positrons [3]. Ones suppose that the X-ray pulsars might be the sources of these positron flows [4]. Thus, there is a significant reason for the investigation of all processes, which take place near a pulsar surface and contribute to the production of positrons. Particularly, in the present article, we examine one relevant process, which makes an influence on the creation of electron-positron fluxes. This is the resonant photoproduction of pair (PPP) by a gamma quantum on a nucleus in the external electromagnetic field. It is a proverbial fact that the presence of an external electromagnetic field causes nonlinear quantum electrodynamics (QED) reactions [5,6] that lead to a cascade production of electron-positron pairs $[7,8]$.

In the present paper, we consider the process of the second order in the fine structure constant. The main feature of such a process is the capability to occur in a resonant way [9-22] when the initial process effectively reduces into two successive processes of the first order in the fine structure constant. 
Herewith, the resonant differential cross section of the considered process significantly exceeds the corresponding one in the absence of an external field. In this article, we develop the theory of several first resonances (with the absorption of one, two, three, and so on photons of an external field) for the resonant PPP by a high-energy gamma quantum in the field of a nucleus and an external field of the X-ray pulsar. We note that this problem has been investigated before for the optical frequency range [23-28]. Meanwhile, in current research, we are interested in the X-ray frequency range that takes place for the $\mathrm{X}$-ray pulsars.

\section{Amplitude of the PPP}

Generally, there are two characteristic parameters in the problem of PPP on a nucleus within an external electromagnetic field. One is the classical relativistically invariant parameter [5]:

$$
\eta_{0}=\frac{e F_{0} t}{m c^{2}}
$$

which numerically equals to the ratio of the field work at a wavelength to the electron rest energy ( $e$ and $m$ are the charge and the electron mass, $F_{0}$ and $t=c / \omega$ are the strength and electromagnetic wavelength, $\omega$ is the wave frequency). Additionally, the second is the quantum multiphoton parameter [29,30] (Bunkin-Fedorov parameter):

$$
\gamma_{0}=\eta_{0} \frac{m v c}{\hbar \omega}
$$

Herein, $v$ is the electron (positron) velocity and $c$ is the speed of light. However, in the current paper, we are interested in the situation, when the electrons (positrons) are scattered by a nucleus at small angles since, as was shown in the paper [23], under the resonant condition all produced particles are ultrarelativistic. According to this fact, the quantum parameter (2) does not appear [31] and the main parameter of multiphoton processes is the classical parameter $\eta_{0}$. Hereafter, we consider the external electromagnetic field as weak, when the following condition is satisfied:

$$
\eta_{0} \ll 1
$$

Such a restriction fulfills near the magnetic poles of $X$-ray pulsar. Taking into account the spectral composition $(1 \div 100 \mathrm{keV})$ [32], the luminosity $\left(10^{38} \div 10^{39} \mathrm{erg} / \mathrm{s}\right)$ [33], and the size $(10 \div 15 \mathrm{~km}$ in radius) [34] of the $X$-ray pulsars, we can conclude that the classical parameter $\eta_{0}$ is of the order of $10^{-2}$. Additionally, it is important to emphasize that we treat the Coulomb interaction of the produced fermions with a nucleus in the first Born approximation. This assumption leads us to the restriction on a nuclear charge $(v / c \gg Z \alpha$, where $Z$ is the nuclear charge, $\alpha$ is the fine structure constant). Due to the fact that X-ray pulsars represent binary accreting systems [33], there is a high concentration of hydrogen and helium atoms within their atmosphere [35], which satisfy the corresponding restriction. Further, we use natural units $\hbar=1, c=1$ throughout this article.

We choose the electromagnetic four-potential of the external field propagating along the $z$ axis in the following form:

$$
A(\varphi)=\left(\frac{F_{0}}{\omega}\right) \cdot g\left(\frac{\varphi}{\omega \tau}\right) \cdot\left(e_{x} \cos \varphi+\delta e_{y} \sin \varphi\right), \quad \varphi=k x=\omega(t-z),
$$

where $k=(\omega, \mathbf{k})$ is the wave four-vector, $\delta$ is the ellipticity parameter of a wave. Additionally, here $e_{x}=\left(0, \mathbf{e}_{x}\right), e_{y}=\left(0, \mathbf{e}_{y}\right)$ are the polarization four-vectors of the wave, particularly $e_{x, y}^{2}=-1,\left(e_{x, y} k\right)=k^{2}=0$. In the expression (3), the function $g(\varphi / \omega \tau)$ is the envelope of our potential. We require $g(0)=1$ and the exponential decreasing of the function $g \rightarrow 0$ when $|\varphi| \gg \omega \tau$. 
Hereinafter, in order to satisfy the condition of quasi-monochromaticity, we assume that the duration of a pulse significantly exceeds the characteristic oscillation time of a wave

$$
\omega \tau \gg 1
$$

We treat the external electromagnetic field of X-ray pulsar as a plane wave, due to that fact we choose the envelope function $g(\varphi / \omega \tau)$ as a function of the invariant variable $\varphi$. Hence, we use the exact solutions of the Dirac equation for an electron and a positron in a field of a plane electromagnetic wave with an arbitrary spectral composition (Volkov solutions [36-38]) to take into account the interaction with an external field of the X-ray pulsar. We use Green's function derived in the following works $[39,40]$ to describe the propagation of the intermediate particle within the external field.

Resonant photoproduction of electron-positron pair on a nucleus in the external electromagnetic field is the second order process in the fine structure constant and it can be depicted by the two Feynman diagrams. In the resonant conditions, the initial process effectively reduces into the two successive processes of the first order in the fine structure constant, as was mentioned above: first is stimulated by the external field Breit-Wheeler process [41,42] and the second is the Mott scattering assisted by the external field [43-45]. We point out that there are two different channels for the considered process. The difference between them is that when the initial gamma quantum produces an electron and a positron one of them is an intermediate particle, which is then scattered by a nucleus. For channel A (Figure 1a), an intermediate particle is an electron and for channel B (Figure 1b), an intermediate particle is a positron.

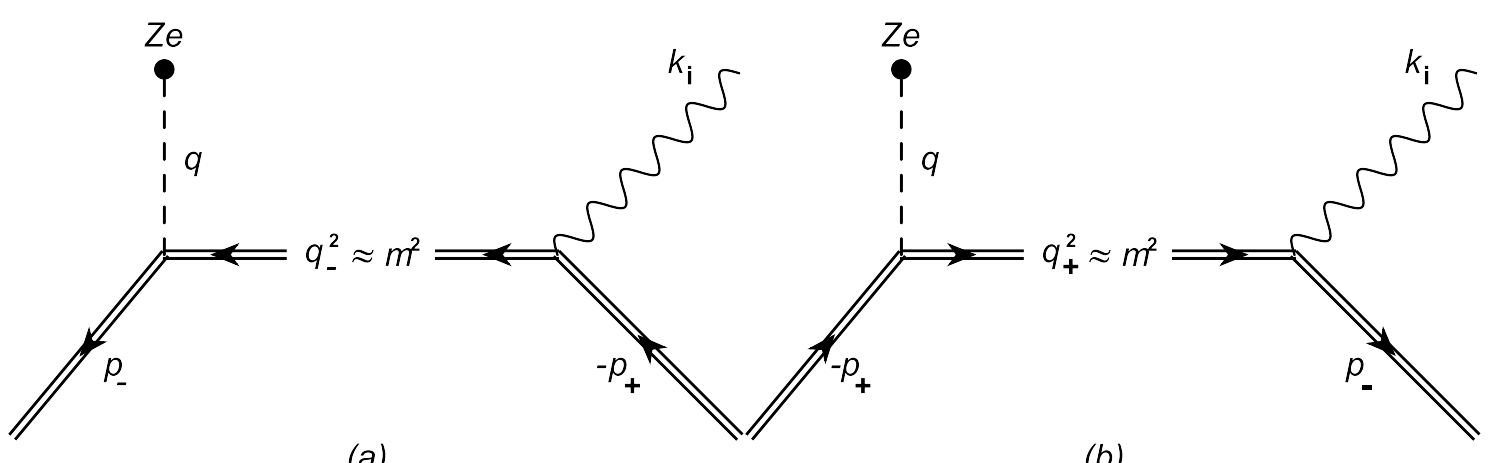

(a)

(b)

Figure 1. Feynman diagrams of the resonant PPP process on a nucleus in the external electromagnetic field. Double incoming and outgoing lines correspond to the Volkov functions of an electron and a positron in the initial and final states, the inner lines correspond to the Green's function of the fermion in the external electromagnetic field. Wavy lines represent the initial gamma quantum. Dashed lines stand for the pseudo photon of recoil. The figures (a) and (b) depict two different channels A and B correspondingly.

When considering the fulfillment of the introduced assumptions (3)-(5) [46,47], we obtained the expression for the amplitude of the PPP process on a nucleus in the external electromagnetic field:

$$
S=\sum_{l=-\infty}^{+\infty} S_{(l)}
$$

Herein, $S_{(l)}$ is a partial amplitude of the process with radiation (absorption) of $|l|$-photons of the external electromagnetic field:

$$
S_{(l)}=i \frac{2 \tau^{2} \omega Z e^{3} \sqrt{\pi}}{\sqrt{2 \omega_{i} E_{-} E_{+}}} \frac{\left(\bar{u}_{p_{-}} B_{(l)} v_{-p_{+}}\right)}{\left[\mathbf{q}^{2}+q_{0}\left(q_{0}-2 q_{z}\right)\right]},
$$


where we denoted:

$$
B_{(l)}=B_{+(l)}+B_{-(l)}, \quad q=p_{-}+p_{+}-k_{i}+l k .
$$

Here, $q=\left(q_{0}, \mathbf{q}\right)$ is a transferred four-momentum, $v_{-p_{+}}$and $\bar{u}_{p_{-}}\left(\bar{u}_{p_{-}}=u_{p_{-}}^{+} \tilde{\gamma}_{0}\right)$ are the Dirac bispinors for a free positron and a free electron, $B_{+(l)}$ and $B_{-(l)}$ are the amplitudes for the channels A and B:

$$
\begin{gathered}
B_{+(l)}=\sum_{r=-\infty}^{\infty} \frac{\pi i}{\left(k q_{-}\right)} \int_{-\infty}^{\infty} d \phi_{1} \int_{-\infty}^{\infty} d \phi_{2} \exp \left(-2 i \beta_{-} \phi_{1}\right) \exp \left[i\left(q_{0} \tau+2 \beta_{-}\right) \phi_{2}\right] \times \\
\times \operatorname{sgn}\left(\phi_{1}-\phi_{2}\right) M_{(l+r)}^{0}\left(p_{-}, q_{-}, \phi_{2}\right)\left(\hat{q}_{-}+m\right)\left[\varepsilon_{\mu}^{*} F_{(-r)}^{\mu}\left(q_{-}, p_{+}, \phi_{1}\right)\right], \\
B_{-(l)}=\sum_{r=-\infty}^{\infty} \frac{\pi i}{\left(k q_{+}\right)} \int_{-\infty}^{\infty} d \phi_{1} \int_{-\infty}^{\infty} d \phi_{2} \exp \left(-2 i \beta_{+} \phi_{1}\right) \exp \left[i\left(q_{0} \tau+2 \beta_{+}\right) \phi_{2}\right] \times \\
\times \operatorname{sgn}\left(\phi_{1}-\phi_{2}\right) M_{(l+r)}^{0}\left(p_{+}, q_{+}, \phi_{2}\right)\left(\hat{q}_{+}+m\right)\left[\varepsilon_{\mu}^{*} F_{-r}^{\mu}\left(q_{+}, p_{-}, \phi_{1}\right)\right], \\
q_{-}=-p_{+}+k_{i}+r k, \quad q_{+}=-p_{-}+k_{i}+r k .
\end{gathered}
$$

Herein, $\beta_{-}$and $\beta_{+}$are the resonant parameters for the channels $\mathrm{A}$ and $\mathrm{B}[46,47]$ :

$$
\beta_{\mp}=\frac{\left(q_{\mp}^{2}-m^{2}\right)}{4\left(k q_{\mp}\right)} \omega \tau .
$$

The notations with hats stand for the dot product of the corresponding four-vector with the Dirac gamma matrices $\tilde{\gamma}^{\mu}$. Besides, $\phi_{n}=\varphi_{n} / \omega \tau(n=1,2)$, $\varepsilon^{\mu}$ and $k_{i}=\left(\omega_{i}, \mathbf{k}_{\mathbf{i}}\right)$ are the polarization four-vector and the four-momentum of the initial gamma quantum correspondingly, $p_{ \pm}=\left(E_{ \pm}, \mathbf{p}_{ \pm}\right)$ are the four-momenta of a positron and an electron, $q_{-}$and $q_{+}$are the four-momenta of an intermediate electron and positron for the channels A and B accordingly (see Figure 1). In the expressions (9) and (10) there is the matrix $M_{(l+r)}^{0}\left(p^{\prime}, p, \phi_{2}\right)$, which under the resonant conditions and with the expression in square brackets in the denominator (7) taken into account represents the amplitude of the intermediate electron (positron) scattering on a nucleus $\left(p \rightarrow p^{\prime}\right)$ with radiation (absorption) of $|l+r|$-photons of the external field. At the same time, $F_{(-r)}^{\mu}\left(p^{\prime}, p, \phi_{1}\right)$ represents the amplitude of an electron-positron pair production (with momenta $p$ and $p^{\prime}$ ) by a gamma quantum $k_{i}$ with absorption of $r$-photons of the external field

$$
\begin{gathered}
M_{(l+r)}^{0}\left(p^{\prime}, p, \phi_{2}\right)=\tilde{\gamma}^{0} L_{l+r}\left(p^{\prime}, p, \phi_{2}\right), \\
F_{(-r)}^{\mu}\left(p^{\prime}, p, \phi_{1}\right)=\tilde{\gamma}^{\mu} L_{-r}\left(p^{\prime}, p, \phi_{1}\right)+b_{p^{\prime} p(-)}^{\mu}\left(\phi_{1}\right) L_{-r-1}+b_{p^{\prime} p(+)}^{\mu}\left(\phi_{1}\right) L_{-r+1} .
\end{gathered}
$$

Here, we introduced matrices $b_{p^{\prime} p( \pm)}^{\mu}$ and special functions $L_{n^{\prime}}\left(p^{\prime}, p, \phi_{n}\right)$, which were studied in details in the following article [48]:

$$
\begin{gathered}
b_{p^{\prime} p( \pm)}^{\mu}\left(\phi_{1}\right)=\eta\left(\phi_{1}\right)\left[\frac{m}{4\left(k p^{\prime}\right)} \hat{e} \hat{k}_{ \pm} \hat{k} \tilde{\gamma}^{\mu}-\frac{m}{4(k p)} \tilde{\gamma}^{\mu} \hat{k} \hat{e}_{ \pm}\right], \\
L_{s}\left(p^{\prime}, p, \phi_{n}\right)=\exp \left(-i s \chi_{p^{\prime} p}\right) J_{s}\left[\gamma_{p^{\prime} p}\left(\phi_{n}\right)\right] .
\end{gathered}
$$

Herein, $J_{s}$ is the Bessel function of an integer index. The parameters $\gamma_{p^{\prime} p}, \chi_{p^{\prime} p}$ and four-vectors $e_{ \pm}$ are defined, as follows:

$$
\begin{gathered}
\gamma_{p^{\prime} p}\left(\phi_{n}\right)=\eta\left(\phi_{n}\right) m \sqrt{-Q_{p^{\prime} p^{\prime}}^{2}}, \quad Q_{p^{\prime} p}=\frac{p^{\prime}}{\left(k p^{\prime}\right)}-\frac{p}{(k p)}, \\
\tan \chi_{p^{\prime} p}=\delta \frac{\left(Q_{p^{\prime} p} e_{y}\right)}{\left(Q_{p^{\prime} p} e_{x}\right)}, \quad e_{ \pm}=e_{x} \pm i \delta e_{y} .
\end{gathered}
$$


In the relations (15)-(17), the parameter $\eta\left(\phi_{n}\right)$ has the following form:

$$
\eta\left(\phi_{n}\right)=\eta_{0} g\left(\phi_{n}\right), \quad n=1,2 .
$$

Henceforth, we shall consider the case of the high-energy initial gamma quanta and the ultrarelativistic energies of the produced electrons and positrons when all particles propagate in a narrow cone along the direction of the initial gamma quantum momentum [23]:

$$
\begin{gathered}
\omega_{i} \gg m, \quad E_{ \pm} \gg m \\
\theta_{i \pm}=\angle\left(\mathbf{k}_{i}, \mathbf{p}_{ \pm}\right) \ll 1, \quad \bar{\theta}_{ \pm}=\angle\left(\mathbf{p}_{+}, \mathbf{p}_{-}\right) \ll 1 \\
\theta_{i}=L\left(\mathbf{k}_{i}, \mathbf{k}\right) \sim 1, \quad \theta_{ \pm}=\angle\left(\mathbf{p}_{ \pm}, \mathbf{k}\right) \sim 1
\end{gathered}
$$

\section{Poles of the PPP Amplitude}

The appearance of resonances in the amplitudes (6)-(10) is connected with the discrete nature of the energy spectrum of the system: electron (positron) + plane electromagnetic wave [49-51]. It follows that due to the approximate fulfillment of the energy-momentum conservation law, four-momentum of the intermediate electron (positron) lies near the mass shell. The following conditions take place for the channels A and B in the resonance (see (9) and (10)):

$$
\left|\beta_{\mp}\right| \lesssim 1 \Rightarrow \frac{\left|q_{\mp}^{2}-m^{2}\right|}{4\left(k q_{\mp}\right)} \lesssim \frac{1}{\omega \tau} \ll 1 .
$$

The resonant parameters $\beta_{\mp}$ (12) for the case of a weak field (5) and the kinematic conditions (20)-(22) have the following form:

$$
\begin{gathered}
\beta_{\mp}=r \frac{\left[4\left(1-x_{ \pm}\right) x_{ \pm} \varepsilon_{r}-4 x_{ \pm}^{2} \delta_{ \pm}^{2}-1\right]}{16 \varepsilon_{r}\left(1-x_{ \pm}\right) x_{ \pm}} \omega \tau, \\
\delta_{ \pm}=\frac{\omega_{i} \theta_{i \pm}}{2 m}, \quad x_{ \pm}=\frac{E_{ \pm}}{\omega_{i}}, \\
\varepsilon_{r}=\frac{\omega_{i}}{\omega_{t h r(r)}}, \quad \omega_{t h r(r)}=\frac{m^{2}}{r \omega \sin \left(\theta_{i} / 2\right)} .
\end{gathered}
$$

Herein, $r=1,2,3 \ldots$ is the number of a resonance (the number of photons, which are absorbed in the stimulated by the external field Breit-Wheeler process), $\omega_{t h r(r)}$ is the threshold energy for the $r$ th-resonance (see (27)-(29)). This energy is defined by the rest energy of an electron, the total energy of absorbed photons, and the angle between the momenta of the initial gamma quantum and the external wave. Within the X-ray range, the threshold energy for the first resonance is of the order of $\omega_{\operatorname{thr}(1)} \sim 10^{2} \mathrm{MeV}$. With an increase in the number of a resonance (absorbed photons), the threshold energy decreases by a factor of $r$ (26). In the expression (26), we denoted the initial gamma quantum energy in the units of threshold energy of $r$ th-resonance as $\varepsilon_{r}$.

Using the expressions for the resonant parameters (24) and the resonant conditions (23), we derived expressions for the particles energy for the channels A (27) and B (28):

$$
\begin{gathered}
x_{+(r)}\left(\delta_{+}^{2}\right) \approx \frac{\varepsilon_{r} \pm \sqrt{\varepsilon_{r}\left(\varepsilon_{r}-1\right)-\delta_{+}^{2}}}{2\left(\varepsilon_{r}+\delta_{+}^{2}\right)}, \quad x_{-(r)}\left(\delta_{+}^{2}\right) \approx 1-x_{+(r)}\left(\delta_{+}^{2}\right), \\
x_{-(r)}\left(\delta_{-}^{2}\right) \approx \frac{\varepsilon_{r} \pm \sqrt{\varepsilon_{r}\left(\varepsilon_{r}-1\right)-\delta_{-}^{2}}}{2\left(\varepsilon_{r}+\delta_{-}^{2}\right)}, \quad x_{+(r)}\left(\delta_{-}^{2}\right) \approx 1-x_{-(r)}\left(\delta_{-}^{2}\right), \quad x_{ \pm(r)}=\frac{E_{ \pm(r)}}{\omega_{i}} .
\end{gathered}
$$


We can conclude that the resonant energies of a positron and an electron for each of the two channels depend on the parameter $\varepsilon_{r}$ and take two different values for a certain positron (channel A) or electron (channel B) outgoing angle (see Figure 2).Herewith, the parameter $\varepsilon_{r}$ has to satisfy the condition:

$$
\varepsilon_{r} \geq 1 \rightarrow \omega_{i} \geq \omega_{t h r(r)}, \quad r=1,2,3, \ldots
$$

(a)

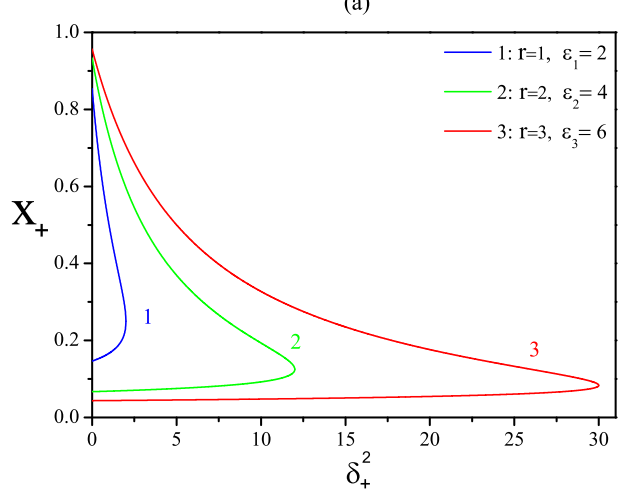

(b)

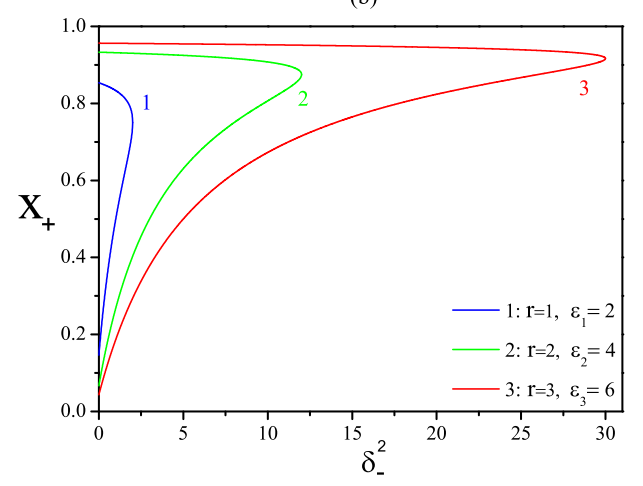

Figure 2. Dependence of the positron resonant energy on the parameter $\delta_{+}^{2}(27)((\mathbf{a})$, for the channel A) and on the parameter $\delta_{-}^{2}(28)\left((\mathbf{b})\right.$, for the channel B) for the case $\omega_{i}>\omega_{t h r(1)}$ and the first three resonances $\left(\omega_{i}=500 \mathrm{MeV}, \omega_{\text {thr }(1)}=250 \mathrm{MeV}\right)$.

It testifies that value $\omega_{t h r(r)}(26)$ is the threshold energy for the initial gamma quantum. This threshold energy decreases with an increase in the number of absorbed photons. From the expressions (27) and (28), we can see that the possible outgoing angles depend on the value of the parameter $\varepsilon_{r}$ and they are enclosed in the interval:

$$
0 \leq \delta_{ \pm}^{2} \leq \delta_{\max }^{2}, \quad \delta_{\max }^{2}=\varepsilon_{r}\left(\varepsilon_{r}-1\right), \quad \varepsilon_{r} \geq 1 .
$$

As we mentioned before in the introduction, there is an anomalous abundance of high-energy positrons in the cosmic rays [3]. Qua attempt to explain this peculiar phenomenon we find it expedient to make calculations for the case when the initial gamma quantum energy extremely exceeds the corresponding threshold energy for the $r$ th-resonance $\left(\varepsilon_{r} \gg 1\right)$. We need to rewrite the expressions (27) and (28) due to the adverted condition and also for the situation of small parameters $\delta_{ \pm}^{2}\left(\varepsilon_{r} \gg \delta_{ \pm}^{2}\right)$. We will see that this assumption is appropriate as the most probable values of the parameters $\delta_{ \pm}^{2}$ satisfy this condition (see Figure 3). After all of the simplifications, we deduced the new expressions for the electron-positron pair energy in resonance:

$$
x_{ \pm(r)}=\left\{\begin{array}{l}
1-\frac{1+4 \delta_{ \pm}^{2}}{4 \varepsilon_{r}} \\
\frac{1}{4 \varepsilon_{r}}
\end{array}\right.
$$




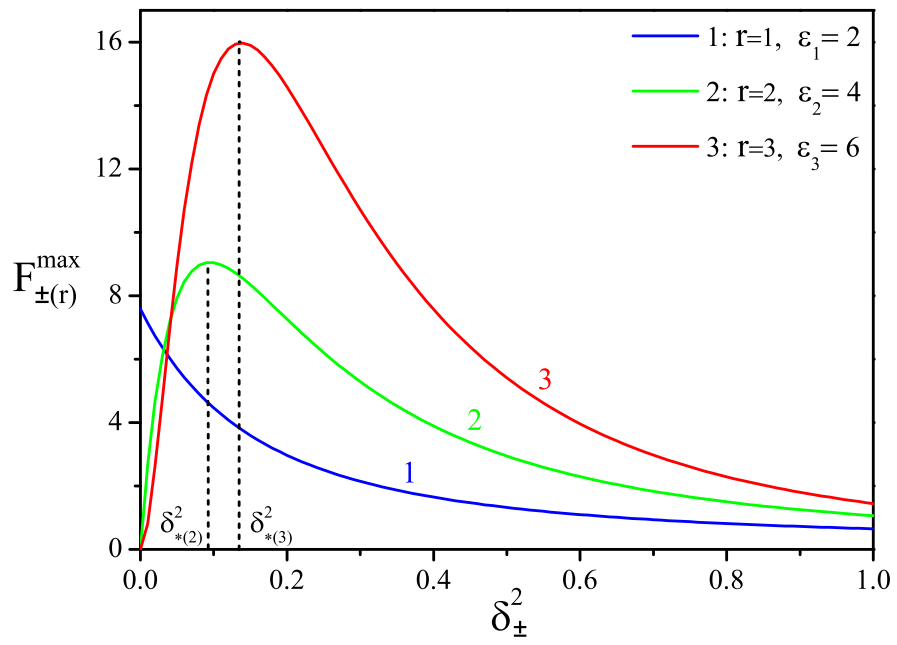

Figure 3. Dependences of the functions $F_{ \pm(r)}^{\max }(56)$ on the parameters $\delta_{ \pm}^{2}$ for the first three resonances with the certain energy of the initial gamma quantum $\omega_{i}=500 \mathrm{MeV}\left(\omega_{i}>\omega_{\text {thr(1) }}=250 \mathrm{MeV}\right)$.

In the obtained expression, top string represents a solution with the sign " + " in front of square root in the expressions (27) and (28), and bottom string stands for the sign "-" correspondingly. As we can see, the top solution almost does not differ from unity, which means that approximately all energy of the initial gamma quantum converts into the positron energy (for channel A) or electron energy (for channel B). In addition, it is important to mention that low-energy solution does not depend on the outgoing angle in the frame of accuracy $\sim 1 / \varepsilon_{r}$.

\section{Resonant Cross Section of the PPP}

We simplify amplitudes of the process (6)-(10) for the case of the resonant kinematics (20)-(22), (27), (28). For the derivation of the resonant cross section, we have to define a certain type of the envelope function (4). Herein, we choose the Gaussian-like form:

$$
g\left(\phi_{1}\right)=\exp \left\{-\left(2 \phi_{1}\right)^{2}\right\}
$$

Carrying out all of the calculations [24] and standard procedures [52], we deduce the expressions for the resonant differential cross section of the PPP on a nucleus in the external electromagnetic field for the channels A and B:

$$
\begin{aligned}
& d \sigma_{+(r)}=d \sigma_{(0)}\left(p_{-}, q_{-}\right) \Psi_{(r)}^{r e s}\left(q_{-}\right) d W_{+(r)}\left(q_{-}, p_{+}\right), \\
& d \sigma_{-(r)}=d \sigma_{(0)}\left(p_{+}, q_{+}\right) \Psi_{(r)}^{r e s}\left(q_{+}\right) d W_{-(r)}\left(q_{+}, p_{-}\right),
\end{aligned}
$$

where we denoted

$$
\begin{gathered}
d W_{+(r)}\left(q_{-}, p_{+}\right)=\frac{\alpha}{\omega_{i} E_{+}} \eta_{0}^{2 r}\left(\frac{r^{r}}{r !}\right)^{2}\left[\frac{u_{+}}{u_{r}}\left(1-\frac{u_{+}}{u_{r}}\right)\right]^{r-1}\left[2 u_{+}-1+2 \frac{u_{+}}{u_{r}}\left(1-\frac{u_{+}}{u_{r}}\right)\right] d^{3} p_{+}, \\
d \sigma^{(0)}\left(p_{-}, q_{-}\right)=2 Z^{2} r_{e}^{2} \frac{\left|\mathbf{p}_{-}\right|}{\left|\mathbf{q}_{-}\right|} \frac{m^{2}\left[m^{2}+p_{-}^{0} q_{-}^{0}+\mathbf{p}_{-} \mathbf{q}_{-}\right]}{\mathbf{q}^{4}} d \Omega_{-}, \quad \mathbf{q}=\mathbf{p}_{-}-\mathbf{q}_{-} .
\end{gathered}
$$

Herein, $d W_{+(r)}\left(q_{-}, p_{+}\right)$is a probability per unit of time of the electron-positron pair photoproduction with the momenta $q_{-}$and $p_{+}$, correspondingly, due to the absorption of $r$-photons of the external field [5]. The expression for $d W_{-(r)}\left(q_{+}, p_{-}\right)$can be obtained from (35) by corresponding 
replacement of the four-momenta. In the expression (35) the relativistically invariant parameters for the channels $\mathrm{A}$ and $\mathrm{B}$ are defined in the following way [5]:

$$
u_{ \pm}=\frac{\left(k k_{i}\right)^{2}}{4\left(k p_{ \pm}\right)\left(k q_{\mp}\right)}, \quad u_{r}=r \frac{\left(k k_{i}\right)}{2 m^{2}} .
$$

We underline that $d \sigma_{(0)}\left(p_{-}, q_{-}\right)$and $d \sigma_{(0)}\left(p_{+}, q_{+}\right)$are the differential cross sections of the intermediate electron (positron) scattering on a nucleus in the absence of radiation (absorption) of photons [30]. Particularly, $d \sigma_{(0)}\left(p_{+}, q_{+}\right)$can be obtained from the expression (36) by replacement of four-momenta: $q_{-} \rightarrow q_{+}, p_{-} \rightarrow p_{+}$. The resonant functions $\Psi_{(r)}^{r e s}\left(q_{ \pm}\right)$have the following forms:

$$
\Psi_{r e s}^{(r)}\left(q_{ \pm}\right)=\frac{m^{2}(\omega \tau)^{2}\left|\mathbf{q}_{ \pm}\right|}{16 \pi^{2} r\left(k q_{ \pm}\right)^{2}} P_{(r)}^{r e s}\left(\beta_{ \pm}\right),
$$

where we introduced a function of resonant profile $P_{(r)}^{r e s}\left(\beta_{ \pm}\right)$, which depends on the resonant parameter $\beta_{ \pm}$and has the following expression:

$$
P_{(r)}^{r e s}\left(\beta_{ \pm}\right)=\exp \left(-\frac{\beta_{ \pm}^{2}}{2 r}\right) \frac{1}{2 \rho} \int_{-\rho}^{\rho} d \phi_{2}\left|\operatorname{erf}\left(2 \sqrt{r} \phi_{2}+\frac{i \beta_{ \pm}}{2 \sqrt{r}}\right)\right|^{2}, \quad \rho=T / \tau .
$$

In the expression (39) $T$ is a some relatively large observation time $(T \gtrsim \tau)$. We are interested in the resonant profile function (39) when resonant parameter $\left|\beta_{ \pm}\right| \ll 1$. In such case, the function of resonant profile $P_{(r)}^{r e s}\left(\beta_{j}\right)$ transforms into the Breit-Wigner formula [23,24,53]:

$$
\begin{gathered}
P_{(r)}^{r e s}\left(\left|\beta_{ \pm}\right| \ll 1\right) \approx \frac{a_{(r)} \Lambda_{ \pm(r)}^{2}}{\left[\left(q_{ \pm}^{2}-m^{2}\right)^{2}+\Lambda_{ \pm(r)}^{2}\right]} \approx P_{\max (r)}^{r e s}=a_{(r)}, \\
\Lambda_{ \pm(r)}=\frac{4 r c_{(r)}\left(k q_{ \pm}\right)}{(\omega \tau)} ; \quad c_{(r)}=\sqrt{\frac{2 a_{(r)}}{r\left(a_{(r)}-2 r b_{(r)}\right)}}, \\
a_{(r)}=\frac{1}{\rho} \int_{0}^{\rho} \operatorname{erf} f^{2}(2 \sqrt{r} \phi) d \phi, \\
b_{(r)}=\frac{1}{\rho \sqrt{\pi r}}\left[\frac{1}{2 \sqrt{2 \pi} r} \operatorname{erf}(2 \sqrt{2 r} \rho)+2 \int_{0}^{\rho} \phi \operatorname{erf}(2 \sqrt{r} \phi) \exp \left(-4 r \phi^{2}\right) d \phi\right] .
\end{gathered}
$$

We want to point out that the function of resonant profile (39) is valid when $\left|\beta_{ \pm}\right| \lesssim 1$. It is important to emphasize that, only for the case $\left|\beta_{ \pm}\right| \ll 1$, the resonant profile function transforms to the Breit-Wigner formula. This fact justifies the condition for applying the phenomenological Breit-Wigner procedure for the case of the plane monochromatic wave [23]. For the first three resonances $a_{(r)} \sim 1, c_{(r)} \sim 1, b_{(r)} \sim 10^{-2}$. We can see from the relation (40) that the maximum of the function is determined by the parameter $a_{(r)}$ (42), which depends on the number of resonance and ratio of the observation time to the duration of the X-ray pulse (parameter $\rho$ ).

In Figure 4, we can see that resonances take place if the resonant parameters $\left|\beta_{ \pm}\right| \lesssim 1$. When $\left|\beta_{ \pm}\right| \gg 1$, the resonant profile function exponentially decreases $[46,47]$. 


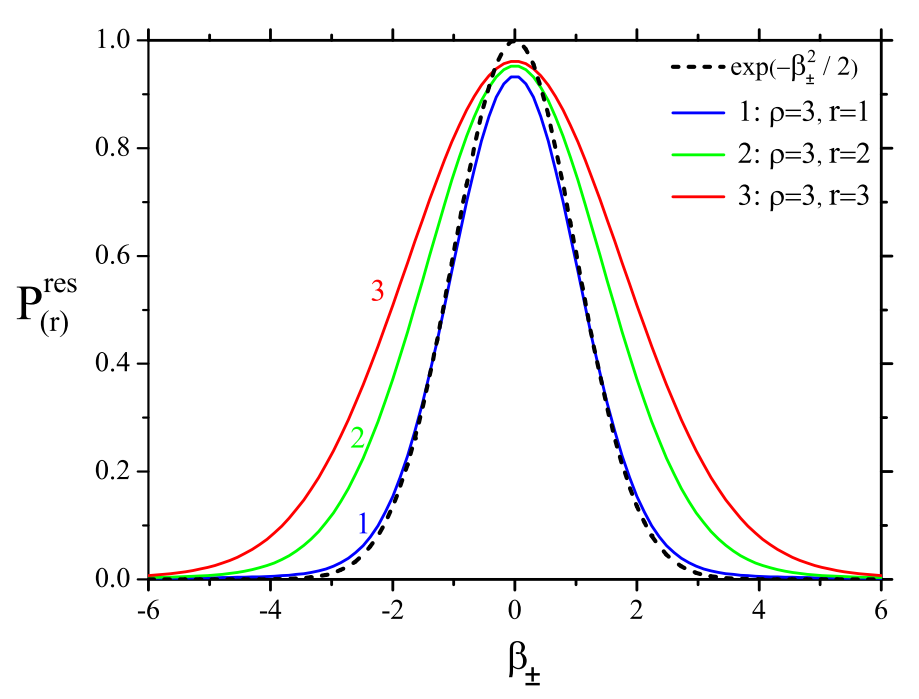

Figure 4. The resonant profile function for the first three resonances.

\section{Analysis of the Resonant Differential cross Section of the PPP by a High-Energy Gamma Quantum}

We rewrite the resonant differential cross section of the PPP (33)-(39) according to the resonant kinematics (20)-(22):

$$
\begin{aligned}
& d \sigma_{+(r)}=\alpha Z^{2} r_{e}^{2} \eta_{0}^{2 r} \frac{r(\omega \tau)^{2}\left(1-x_{+(r)}\right) x_{+(r)}}{8 \pi d_{+(r)}^{2}} \frac{K_{+(r)}}{\varepsilon_{r}^{2}} P_{(r)}^{r e s}\left(\beta_{-}\right) d \delta_{+}^{2} d \delta_{-}^{2} d x_{+(r)} d \varphi, \\
& d \sigma_{-(r)}=\alpha Z^{2} r_{e}^{2} \eta_{0}^{2 r} \frac{r(\omega \tau)^{2}\left(1-x_{-(r)}\right) x_{-(r)}}{8 \pi d_{-(r)}^{2}} \frac{K_{-(r)}}{\varepsilon_{r}^{2}} P_{(r)}^{r e s}\left(\beta_{+}\right) d \delta_{+}^{2} d \delta_{-}^{2} d x_{-(r)} d \varphi .
\end{aligned}
$$

Herein, $\varphi$ is an angle between the planes $\left(\mathbf{k}_{i} \mathbf{p}_{+}\right)$and $\left(\mathbf{k}_{i} \mathbf{p}_{-}\right)$. The resonant profile functions $P_{(r)}^{r r s}\left(\beta_{\mp}\right)$ are determined by the expression (39), where the resonant parameters are given by the relation (24). Functions $K_{ \pm(r)}$ are defined by the following expression:

$$
K_{ \pm(r)}=\left(\frac{r^{r}}{r !}\right)^{2}\left[\frac{4 x_{ \pm(r)}^{2} \delta_{ \pm}^{2}}{\left(1+4 x_{ \pm(r)}^{2} \delta_{ \pm}^{2}\right)^{2}}\right]^{r-1}\left[\frac{16 x_{ \pm(r)}^{2} \delta_{ \pm}^{2}}{\left(1+4 x_{ \pm(r)}^{2} \delta_{ \pm}^{2}\right)^{2}}+\left(\frac{x_{ \pm(r)}}{1-x_{ \pm(r)}}+\frac{1-x_{ \pm(r)}}{x_{ \pm(r)}}\right)\right]
$$

The values $d_{ \pm(r)}$ are defined by the square of the transferred momentum:

$$
d_{ \pm(r)}=d_{0}\left(x_{ \pm(r)}\right)+\left(\frac{m}{2 \omega_{i}}\right)^{2}\left(g_{0}^{2}\left(x_{ \pm(r)}\right)+\frac{4 \varepsilon_{r}}{\sin \left(\theta_{i} / 2\right)}\left[4 \varepsilon_{r}-g_{0}^{2}\left(x_{ \pm(r)}\right)\right]\right)
$$

where

$$
\begin{gathered}
d_{0}\left(x_{ \pm(r)}\right)=\tilde{\delta}_{+}^{2}+\tilde{\delta}_{-}^{2}+2 \tilde{\delta}_{+} \tilde{\delta}_{-} \cos \varphi \\
g_{0}\left(x_{+(r)}\right)=\frac{1+\tilde{\delta}_{+}^{2}}{x_{+(r)}}+\frac{1+\tilde{\delta}_{-}^{2}}{1-x_{+(r)}}, \quad g_{0}\left(x_{-(r)}\right)=\frac{1+\tilde{\delta}_{-}^{2}}{x_{-(r)}}+\frac{1+\tilde{\delta}_{+}^{2}}{1-x_{-(r)}} \\
\tilde{\delta}_{ \pm}=2 x_{ \pm(r)} \delta_{ \pm} .
\end{gathered}
$$


It is important to emphasize that in the differential cross Sections (44)-(45), (47), were introduced the small corrections that are proportional to $\sim\left(m / \omega_{i}\right)^{2} \ll 1$. These corrections make a dominant contribution to the corresponding differential cross sections under the conditions:

$$
|\varphi-\pi| \lesssim \frac{m}{\omega_{i}}, \quad\left|\tilde{\delta}_{+}-\tilde{\delta}_{-}\right| \lesssim \frac{m}{\omega_{i}} .
$$

These conditions lead to $D_{0}\left(x_{ \pm}\right) \rightarrow 0, d_{0}\left(x_{ \pm}\right) \rightarrow 0$ and, consequently, the corresponding differential cross section has a sharp peak $[23,24]$.

We integrate the resonant differential cross section with respect to the parameter $\delta_{-}^{2}$ (for the channel A) and the parameter $\delta_{+}^{2}$ (for the channel B):

$$
\begin{aligned}
d \sigma_{+(r)}^{\max }= & \left(\alpha r_{e}^{2} Z^{2}\right) \Phi_{+(r)}^{\max } d x_{+(r)} d \delta_{+}^{2}, \\
d \sigma_{-(r)}^{\max }= & \left(\alpha r_{e}^{2} Z^{2}\right) \Phi_{-(r)}^{\max } d x_{-(r)} d \delta_{-}^{2}, \\
g_{r}= & \left(\frac{\omega_{t h r(r)}}{m}\right)^{2}\left(\eta_{0}^{r} \omega \tau\right)^{2}, \\
& \Phi_{ \pm(r)}^{\max }=g_{r} F_{ \pm(r)^{\prime}}^{\max } \\
F_{ \pm(r)}^{\max }= & \frac{r a_{(r)}}{\varepsilon_{r}^{2}}\left(\frac{x_{ \pm(r)}}{1-x_{ \pm(r)}}\right) K_{ \pm(r)} .
\end{aligned}
$$

The relation (52) defines the maximum (when $\left|\beta_{-}\right| \ll 1,\left|\beta_{+}\right| \ll 1$ ) resonant differential cross section of the PPP with simultaneous registration of the positron outgoing angle and the positron energy (irrespective of the electron outgoing angles). Meanwhile, the relation (53) defines the resonant differential cross section of the PPP with simultaneous registration of the outgoing electron angle and the electron energy (irrespective of the positron outgoing angles). We underline that the function $g_{r}(54)$ is the same for both channels. Here, function $g_{r}$ essentially depends on the field intensity and also on the resonance number. We represent numerical assessment in Table 1 . We underline that the

\begin{tabular}{|c|c|c|c|c|c|}
\hline \multicolumn{6}{|c|}{$\omega_{i}=500 \mathrm{MeV}, \quad \omega_{t h r(1)}=250 \mathrm{MeV}$} \\
\hline$r$ & Channel & $\delta_{ \pm(r)}^{2}$ & $E_{+}, \mathrm{MeV}$ & $E_{-}, \mathrm{MeV}$ & $\Phi_{ \pm(r)}^{\max }(\eta=0.01)$ \\
\hline \multirow{2}{*}{1} & A & $0 \leq \delta_{+}^{2} \leq 2$ & $73.2 \leq E_{+} \leq 426.8$ & $E_{-}=\left(500-E_{+}\right)$ & \multirow{2}{*}{$7.3 \cdot 10^{8} \leq \Phi_{ \pm(1)}^{\max } \leq 1.8 \cdot 10^{10}$} \\
\hline & B & $0 \leq \delta_{-}^{2} \leq 2$ & $E_{+}=\left(500-E_{-}\right)$ & $73.2 \leq E_{-} \leq 426.8$ & \\
\hline \multirow{2}{*}{2} & $\mathrm{~A}$ & $\delta_{+(2)}^{2}=\delta_{*(2)}^{2}=0.095$ & 454.89 & 45.11 & \multirow{2}{*}{$\Phi_{ \pm(2)}^{\max } \approx 5.42 \cdot 10^{5}$} \\
\hline & B & $\delta_{-(2)}^{2}=\delta_{*(2)}^{2}=0.095$ & 45.11 & 454.89 & \\
\hline \multirow{2}{*}{3} & A & $\delta_{+(3)}^{2}=\delta_{*(3)}^{2}=0.138$ & 466.98 & 33.02 & \multirow{2}{*}{$\Phi_{ \pm(3)}^{\max } \approx 42.49$} \\
\hline & B & $\delta_{-(3)}^{2}=\delta_{*(3)}^{2}=0.138$ & 33.02 & 466.98 & \\
\hline
\end{tabular}
obtained expressions (52)-(56), which define the resonant differential cross section within the pulsed wave field, differ from the corresponding expressions in the field of a plane monochromatic wave (see, for example, [23]).

Table 1. The most probable values of the electron-positron pair energies and the corresponding resonant differential cross sections for the case $\varepsilon_{r} \sim 1$.

Now we transform the expressions (56) for the case $\varepsilon_{r} \gg 1$ and the high-energy solutions in (31):

$$
F_{ \pm(r)}^{\max }=r a_{(r)}\left(\frac{r^{r}}{r !}\right)^{2} \frac{16}{\left(1+4 \delta_{ \pm}^{2}\right)^{2}}\left[\frac{4 \delta_{ \pm}^{2}}{\left(1+4 \delta_{ \pm}^{2}\right)^{2}}\right]^{r-1}
$$


We derived that the angular distributions do not depend on the parameter $\varepsilon_{r}$ and depend only on the corresponding outgoing angle. The qualitative behavior remains the same (see Figure 5). We represent the corresponding numerical estimates for the case $\varepsilon_{r} \gg 1$ in Table 2. As we can see from the Table 2, for channel A we have the situation when almost all initial gamma quantum energy converts into the positron energy. Therefore, when the energy of the initial gamma quantum has the order of $10^{2} \mathrm{GeV}$ the production of high-energy ultrarelativistic positrons takes place. Consequently, we can conclude that the considered process may be one of the reasons for the presence of an anomalous amount of ultrarelativistic positrons in cosmic rays. We note that the case of extremely large energies of the initial gamma quanta $\left(\varepsilon_{r} \gg 1\right)$ has not been considered before (see, for example $\left.[23,24]\right)$. We also accentuate that in contrast to the paper [23], where only first resonance was considered, in the present article we considered several first resonances. Herewith, we deduced that, with an increase in a number of resonance (number of absorbed wave photons), the resonant differential cross sections decrease; nevertheless, their magnitude is still large enough (see Tables 1 and 2) for the first, second, and third resonances.

Table 2. The most probable values of the electron-positron pair energies and the corresponding resonant differential cross sections for the the case $\varepsilon_{r} \gg 1$.

\begin{tabular}{|c|c|c|c|c|c|}
\hline \multicolumn{6}{|c|}{$\omega_{i}=100 \mathrm{GeV}, \quad \omega_{\operatorname{thr}(1)}=100 \mathrm{MeV}$} \\
\hline$r$ & Channel & $\delta_{ \pm(r)}^{2}$ & $E_{+}, \mathrm{GeV}$ & $E_{-}, \mathrm{GeV}$ & $\Phi_{ \pm(r)}^{\max }(\eta=0.01)$ \\
\hline \multirow{2}{*}{1} & A & $0 \leq \delta_{+}^{2} \leq 9.99 * 10^{3}$ & $0.025 \leq E_{+} \leq 99.975$ & $E_{-}=\left(100-E_{+}\right)$ & \multirow{2}{*}{$3.3 \cdot 10^{2} \leq \Phi_{ \pm(1)}^{\max } \leq 5.3 \cdot 10^{5}$} \\
\hline & B & $0 \leq \delta_{-}^{2} \leq 9.99 * 10^{3}$ & $E_{+}=\left(100-E_{-}\right)$ & $0.025 \leq E_{-} \leq 99.975$ & \\
\hline \multirow{2}{*}{2} & A & $\delta_{+(2)}^{2}=\delta_{*(2)}^{2}=0.083$ & 99.98 & 0.02 & \multirow{2}{*}{$\Phi_{ \pm(2)}^{\max } \approx 1.17 \cdot 10^{5}$} \\
\hline & B & $\delta_{-(2)}^{2}=\delta_{*(2)}^{2}=0.083$ & 0.02 & 99.98 & \\
\hline \multirow{2}{*}{3} & A & $\delta_{+(3)}^{2}=\delta_{*(3)}^{2}=0.125$ & 99.99 & 0.01 & \multirow{2}{*}{$\Phi_{ \pm(3)}^{\max } \approx 4.87$} \\
\hline & B & $\delta_{-(3)}^{2}=\delta_{*(3)}^{2}=0.125$ & 0.01 & 99.99 & \\
\hline
\end{tabular}

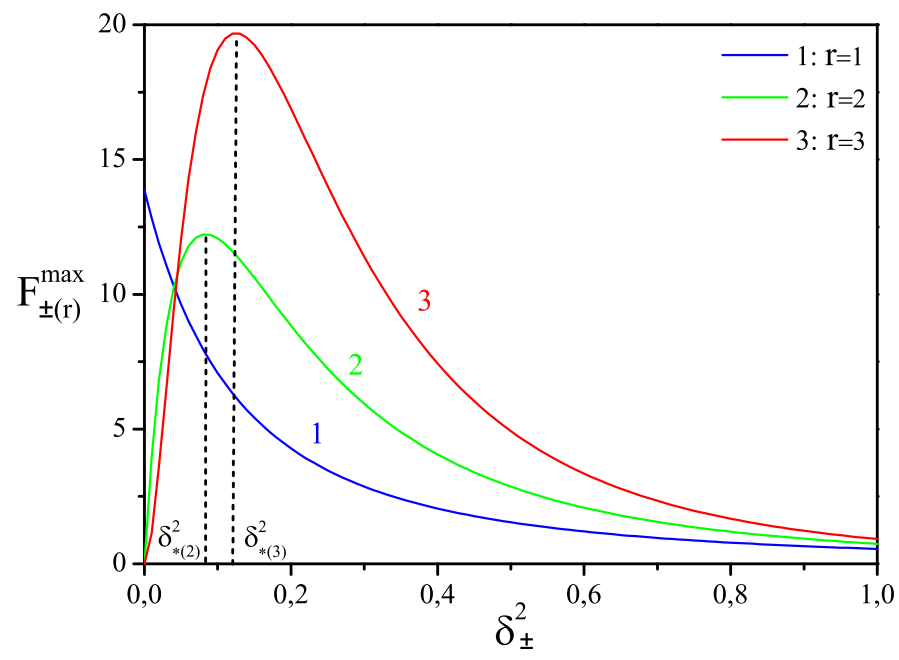

Figure 5. Dependences of the functions $F_{ \pm(r)}^{\max }(57)$ on the parameters $\delta_{ \pm}^{2}$ for the first three resonances and for the case $\varepsilon_{r} \gg 1$.

We point out that, in the presented work, the model of a plane quasi-monochromatic wave was proposed. In fact, the X-ray field of pulsar is inhomogeneous in space and time. Nonetheless, modeling of the X-ray field by plane pulsed field gives us the opportunity to derive analytical expressions for the resonant cross section and possibility to obtain a qualitative assessment of the resonant cross section magnitude near the pulsar surface. We also underline that the influence of 
the magnetic field on the considering process was not studied in the present paper. This is explained by the fact that we consider the X-ray field in the region of the magnetic poles, where, at some distance, the magnetic field does not affect the considering process as much as X-ray field. We emphasize that, for the correct solution of the problem, it is necessary to take into account the influence of the magnetic field on the considering process.

\section{Conclusions}

We investigated the resonant PPP on a nucleus in the field of an external electromagnetic field. This is one of the processes, which contributes to the formation of electron-positron fluxes near the surface of the X-ray pulsar. Additionally, we can now formulate the main results.

There is the threshold energy of the process, which, for the X-ray range, is of the order of $10^{2} \mathrm{MeV}$. The value of the threshold energy significantly depends on the number of absorbed photons of an external field and the angle between momenta of the initial gamma quantum and an external plane wave. We underline that we investigated the situation when all particles propagate in a narrow cone at a large angle relative to wave propagation. The energy of produced particles strongly depends on the outgoing angles (parameters $\delta_{+}^{2}$ and $\delta_{-}^{2}$ ). For channel $\mathrm{A}$, the energy of the pair depends on the positron outgoing angle, for channel B, on the contrary, depends on the electron outgoing angle. Moreover, there are two different values of energy for each (except one) outgoing angle.

We obtained the distribution of the resonant cross section over the outgoing angles of a positron (channel A) or an electron (channel B) for the higher resonances $(r=2,3, \ldots)$, and we can conclude that there are clearly defined maxima in contrast to the corresponding distribution of the first resonance. The most probable positron and electron energies essentially differ from each other as for the frame of the same resonance, so for the different resonances.

It is important to emphasize that the derived resonant cross section has an extremely large value of magnitude (in units $\alpha Z^{2} r_{e}^{2}$ ). However, for our calculations, we made the assumption that an external electromagnetic field has a pulsed character. This fact made an influence on the magnitude of the resonant cross section and reduced it. Indeed, the resonant cross section of PPP on a nucleus in the external electromagnetic field of X-ray pulsar might be even greater. From our estimates, we can conclude that the considered process may contribute to the production of ultrarelativistic positrons, which is able to lead to an anomalous abundance of ultrarelativistic positrons in the cosmic rays.

Author Contributions: Conceptualization, S.P.R.; methodology, S.P.R. and V.V.D.; software, N.R.L.; validation, S.P.R., V.V.D. and N.R.L.; formal analysis, S.P.R. and N.R.L.; investigation, S.P.R., V.V.D. and N.R.L.; data curation, S.P.R. and V.V.D.; writing-original draft preparation, N.R.L.; writing—review and editing, S.P.R. and N.R.L.; supervision, S.P.R. and V.V.D. All authors have read and agreed to the published version of the manuscript.

Funding: This research received no external funding.

Conflicts of Interest: The authors declare no conflict of interest.

\section{References}

1. Khlopov, M. Cosmoparticle physics: The universe as a laboratory of elementary particles. Astron. Rep. 2015, 59, 494-502. [CrossRef]

2. Goldreich, P.; Julian, W.H. Pulsar Electrodynamics. Astrophys. J. 1969, 157, 869. [CrossRef]

3. Adriani, O.; Barbarino, G.; Bazilevskaya, G.A.; Bellotti, R.; Boezio, M.; Bogomolov, E.A.; Bonechi, L.; Bongi, M.; Bonvicini, V.; Bottai, S.; et al. An anomalous positron abundance in cosmic rays with energies 1.5-100 GeV. Nature 2009, 458, 607-609. [CrossRef]

4. Hooper, D.; Blasi, P.; Serpico, P.D. Pulsars as the sources of high energy cosmic ray positrons. J. Cosmol. Astropart. Phys. 2009, 2009, 025-038. [CrossRef]

5. Ritus, V.I.; Nikishov, A.I. Quantum Electrodynamics Phenomena in the Intense Field. In Trudy FIAN; Nauka: Moscow, Russia, 1979; Volume 111.

6. Kuznetsov, A.; Mikheev, N. Electroweak Processes in External Electromagnetic Fields, 1st ed.; Springer Tracts in Modern Physics; Springer: Berlin/Heidelberg, Germany, 2003. 
7. Jones, P.B. Pair production in the pulsar magnetosphere. Astrophys. J. 1979, 228, 536-540. [CrossRef]

8. Timokhin, A.N. Time-dependent pair cascades in magnetospheres of neutron stars-I. Dynamics of the polar cap cascade with no particle supply from the neutron star surface. Mon. Not. R. Astron. Soc. 2010, 408, 2092-2114. [CrossRef]

9. Lebed', A.A.; Padusenko, E.A.; Roshchupkin, S.P.; Dubov, V.V. Resonant parametric interference effect in spontaneous bremsstrahlung of an electron in the field of a nucleus and two pulsed laser waves. Phys. Rev. A 2018, 97, 043404. [CrossRef]

10. Roshchupkin, S.P. Resonance effects with the photoproduction of electron-positron pairs in the field of a plane electromagnetic wave. Sov. Phys. J. 1983, 26, 683-686. [CrossRef]

11. Roshchupkin, S.P. Resonant electron-electron scattering in the field of a light wave: The general relativistic case. Laser Phys. 1994, 4, 139-147.

12. Roshchupkin, S.P. Resonant effects in collisions of relativistic electrons in the field of a light wave. Laser Phys. 1996, 6, 837-858.

13. Nedoreshta, V.N.; Roshchupkin, S.P.; Voroshilo, A.I. Resonance of the exchange amplitude of a photon by an electron scattering in a pulsed laser field. Phys. Rev. A 2015, 91, 062110. [CrossRef]

14. Voroshilo, A.I.; Roshchupkin, S.P.; Denisenko, O.I. Resonance of exchange amplitude of Compton effect in the circularly polarized laser field. Eur. Phys. J. D 2007, 41, 433-440. [CrossRef]

15. Padusenko, E.A.; Roshchupkin, S.P. Resonant scattering of a lepton by a lepton in the pulsed light field. Laser Phys. 2010, 20, 2080-2091. [CrossRef]

16. Voroshilo, A.I.; Roshchupkin, S.P. Resonant scattering of a photon by an electron in the field of a circularly polarized electromagnetic wave. Laser Phys. Lett. 2005, 2, 184-189. [CrossRef]

17. Nedoreshta, V.N.; Voroshilo, A.I.; Roshchupkin, S.P. Resonant scattering of a photon by an electron in the moderately-strong-pulsed laser field. Phys. Rev. A 2013, 88, 052109. [CrossRef]

18. Nedoreshta, V.N.; Voroshilo, A.I.; Roshchupkin, S.P. Resonant scattering of an electron by a muon in the field of light wave. Eur. Phys. J. D 2008, 48, 451. [CrossRef]

19. Voroshilo, A.I.; Roshchupkin, S.P.; Nedoreshta, V.N. Resonant scattering of photon by electron in the presence of the pulsed laser field. Laser Phys. 2011, 21, 1675. [CrossRef]

20. Lebed', A.A.; Padusenko, E.A.; Roshchupkin, S.P. Resonant scattering of ultrarelativistic electrons in the strong field of a pulsed laser wave. Laser Phys. 2015, 26, 025302. [CrossRef]

21. Lebed', A.A.; Roshchupkin, S.P. Resonant spontaneous bremsstrahlung by an electron scattered by a nucleus in the field of a pulsed light wave. Phys. Rev. A 2010, 81, 033413. [CrossRef]

22. Voroshilo, A.I.; Roshchupkin, S.P.; Nedoreshta, V.N. Resonant two-photon annihilation of an electron-positron pair in a pulsed electromagnetic wave. Phys. Rev. A 2016, 94, 032128. [CrossRef]

23. Larin, N.R.; Dubov, V.V.; Roshchupkin, S.P. Resonant photoproduction of high-energy electron-positron pairs in the field of a nucleus and a weak electromagnetic wave. Phys. Rev. A 2019, 100, 052502. [CrossRef]

24. Roshchupkin, S.P.; Larin, N.R.; Dubov, V.V. Resonant effect at the ultrarelativistic electron-positron pairs production by gamma quanta in the field of a nucleus and a pulsed light wave. arXiv 2020, arXiv:2004.01530.

25. Larin, N.R.; Dubov, V.V.; Roschupkin, S.P. Resonant laser-assisted process of the ultrarelativistic electron-positron pair creation by a gamma quantum in the nucleus field. AIP Conf. Proc. 2019, 2179, 020016. [CrossRef]

26. Larin, N.R.; Dubov, V.V.; Roshchupkin, S.P. Resonant production of electron-positron pairs by a hard gamma-ray on a nucleus in an external electromagnetic field. Mod. Phys. Lett. A 2020, 35, 2040025. [CrossRef]

27. Roshchupkin, S.P.; Dubov, V.V.; Larin, N.R. Resonant Production of an Ultrarelativistic Electron-Positron Pair by a Gamma Quantum in the Field of a Nucleus and a Laser Wave. In Proceedings of the 2019 PhotonIcs Electromagnetics Research Symposium—Spring (PIERS-Spring), Rome, Italy, 17-20 June 2019; pp. 4214-4219.

28. Roshchupkin, S.P.; Dubov, V.V.; Larin, N.R.; Doroshenko, D.V. New aspects of resonant effects in laser-modified Quantum Electrodynamics processes: (Invited). In Proceedings of the 2019 IEEE 8th International Conference on Advanced Optoelectronics and Lasers (CAOL), Sozopol, Bulgaria, 6-8 September 2019; pp. 192-197.

29. Bunkin, F.V.; Fedorov, M.V. Bremsstrahlung in a Strong Radiation Field. J. Exp. Theor. Phys 1966, $22,844-847$.

30. Fedorov, M.V. An Electron in a Strong Light Field; Nauka: Moscow, Russia, 1991. (In Russian) 
31. Lebed', A.A. Electron-nucleus scattering at small angles in the field of a pulsed laser wave. Laser Phys. Lett. 2016, 13, 045401. [CrossRef]

32. Trümper, J.E.; Zezas, A.; Ertan, Ü.; Kylafis, N.D. The energy spectrum of anomalous X-ray pulsars and soft gamma-ray repeaters. Astron. Astrophys. 2010, 518, A46. [CrossRef]

33. Walter, R.; Ferrigno, C. X-ray Pulsars. In Handbook of Supernovae; Alsabti, A.W., Murdin, P., Eds.; Springer International Publishing: Cham, Switzerland, 2017; pp. 1385-1399. [CrossRef]

34. Bauswein, A.; Just, O.; Janka, H.T.; Stergioulas, N. Neutron-star Radius Constraints from GW170817 and Future Detections. Astrophys. J. 2017, 850, L34. [CrossRef]

35. Servillat, M.; Heinke, C.O.; Ho, W.C.G.; Grindlay, J.E.; Hong, J.; van den Berg, M.; Bogdanov, S. Neutron star atmosphere composition: The quiescent, low-mass X-ray binary in the globular cluster M28. Mon. Not. R. Astron. Soc. 2012, 423, 1556-1561. [CrossRef]

36. Volkov, D. On a class of solutions of the Dirac equation. Z. Phys. 1935, 94, 250-260.

37. Wang, H.; Zhong, M.; Gan, L.F. Orthonormality of Volkov Solutions and the Sufficient Condition. Commun. Theor. Phys. 2019, 71, 1179. [CrossRef]

38. Zakowicz, S. Square-integrable wave packets from the Volkov solutions. J. Math. Phys. 2005, 46, 032304. [CrossRef]

39. Brown, L.S.; Kibble, T.W.B. Interaction of Intense Laser Beams with Electrons. Phys. Rev. 1964, 133, A705-A719. [CrossRef]

40. Schwinger, J. On Gauge Invariance and Vacuum Polarization. Phys. Rev. 1951, 82, 664-679. [CrossRef]

41. Krajewska, K.; Kamiński, J.Z. Breit-Wheeler process in intense short laser pulses. Phys. Rev. A 2012, 86, 052104. [CrossRef]

42. Titov, A.I.; Takabe, H.; Kämpfer, B.; Hosaka, A. Enhanced Subthreshold $e^{+} e^{-}$Production in Short Laser Pulses. Phys. Rev. Lett. 2012, 108, 240406. [CrossRef]

43. Li, S.M.; Berakdar, J.; Chen, J.; Zhou, Z.F. Mott scattering in the presence of a linearly polarized laser field. Phys. Rev. A 2003, 67, 063409. [CrossRef]

44. Attaourti, Y.; Manaut, B. Comment on "Mott scattering in strong laser fields". Phys. Rev. A 2003, 68, 067401. [CrossRef]

45. Szymanowski, C.; Véniard, V.; Taïeb, R.; Maquet, A.; Keitel, C.H. Mott scattering in strong laser fields. Phys. Rev. A 1997, 56, 3846-3859. [CrossRef]

46. Roshchupkin, S.P.; Lebed', A.A.; Padusenko, E.A.; Voroshilo, A.I. Resonant effects of quantum electrodynamics in the pulsed light field. In Quantum Optics and Laser Experiments; Lyagushyn, S., Ed.; Intech: Croatia, Rijeka, 2012; Chapter 6, pp. 107-156.

47. Roshchupkin, S.P.; Lebed', A.A. Effects of Quantum Electrodynamics in the Strong Pulsed Laser Fields; Naukova Dumka: Kiev, Ukraine, 2013.

48. Roshchupkin, S.P.; Tsybul'nik, V.A.; Chmirev, A.N. Probability of multiphoton processes in phenomena of a quantum electrodynamics in a strong light field. Laser Phys. 2000, 10, 1231-1248.

49. Oleinik, V. Resonance Effects in the Field of an Intense Laser Beam. J. Exp. Theor. Phys. 1967, 25, 697.

50. Oleinik, V. Resonance Effects in the Field of an Intense Laser Ray. J. Exp. Theor. Phys. 1968, 26, 1132.

51. Krajewska, K. Electron-positron pair creation and Oleinik resonances. Laser Phys. 2011, 21, $1275-1287$. [CrossRef]

52. Berestetskii, V.; Pitaevskii, L.; Lifshitz, E. Quantum Electrodynamics; Number 4; Elsevier Science: Oxford, UK, 2012.

53. Breit, G.; Wigner, E. Capture of Slow Neutrons. Phys. Rev. 1936, 49, 519-531. [CrossRef]

(c) 2020 by the authors. Licensee MDPI, Basel, Switzerland. This article is an open access article distributed under the terms and conditions of the Creative Commons Attribution (CC BY) license (http://creativecommons.org/licenses/by/4.0/). 